\title{
ASPECTOS TÉCNICOS DE LA DIGITALIZACIÓN DE FONDOS AUDIOVISUALES
}

David González-Ruiz, Miquel Térmens y Mireia Ribera
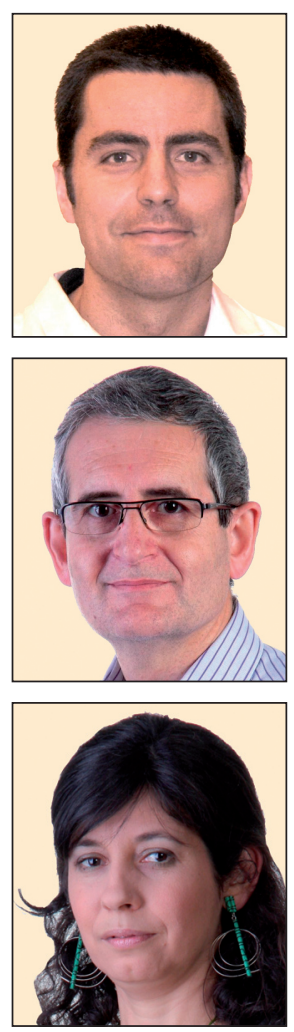

David González-Ruiz es licenciado en historia por la Universitat de Barcelona (UB), licenciado en documentación por la Universitat Autònoma de Barcelona (UAB) y Master en Gestión de Contenidos Digitales por la UB. Trabaja como técnico superior de archivos en la Sección de Imagen y Sonido del Archivo Histórico de Sabadell y es asesor en materia de gestión documental del Club Natación Sabadell.

Arxiu Històric de Sabadell Industria, 32. 08202 Sabadell, España dgonzalez@ajsabadell.cat

Miquel Térmens, doctor en documentación, es profesor del Departamento de Biblioteconomía y Documentación de la Universitat de Barcelona (UB), donde coordina el Máster en Gestión de Contenidos Digitales de la UB-UPF. Es especialista en gestión de la biblioteca digital y en preservación digital, su actual línea de investigación.

Univ. de Barcelona, Fac. de Biblioteconomia i Documentació Melcior de Palau, 140. 08014 Barcelona, España termens@ub.edu

Mireia Ribera es doctora en documentación e ingeniera informática. Es profesora del Departamento de Biblioteconomía y Documentación de la Universitat de Barcelona (UB). Trabaja en el área de la accesibilidad digital y es especialista en formatos de documentos digitales. Recientemente ha colaborado y dirigido diversos estudios sobre vídeo digital.

Univ. de Barcelona, Fac. de Biblioteconomia i Documentació Melcior de Palau, 140. 08014 Barcelona, España ribera@ub.edu

\section{Resumen}

El patrimonio audiovisual de archivos, bibliotecas y museos se encuentra en peligro debido al deterioro de las grabaciones en soportes magnéticos. Se presentan las alteraciones que pueden sufrir las cintas de vídeo, así como las dificultades que representa la obsolescencia de los aparatos necesarios para su lectura. Se considera que la mejor vía de preservación de las cintas de vídeo es su digitalización. Este paso no es fácil dada la complejidad de los formatos de archivos de vídeo digital, incluyendo los contenedores multimedia y los estándares de compresión de vídeo y de audio. Por ello la elección técnica tiene que estar estrechamente ligada a las necesidades de cada servicio. Esta aproximación se ilustra con el caso de los fondos videográficos de las televisiones locales.

\section{Palabras clave}

Archivos audiovisuales, Preservación digital, Digitalización, Vídeo digital, Formatos de archivo, Compresión de datos.

\section{Title: Technical aspects of the digitization of audiovisual holdings}

\begin{abstract}
The audiovisual holdings of archives, libraries and museums are in danger due to the degradation of analogical content recorded on tapes. The article lists the possible damages suffered by videotapes as well as the problems posed by the technical obsolescence of equipment needed to read them. There is an international consensus that digitalization is the best way to preserve videotapes. Digitization is not an easy process considering the complexity of video's digital file formats, which include rich multimedia wrappers and multiple standards for compressing video and audio. Technical decisions should specifically address the needs of each different service, which is illustrated with a case study on audiovisual recordings from local televisions in Spain.
\end{abstract}




\section{Keywords}

Audiovisual archives, Digital preservation, Digitization, Digital video, File formats, Data compression.

González-Ruiz, David; Térmens, Miquel; Ribera, Mireia. "Aspectos técnicos de la digitalización de fondos audiovisuales". El profesional de la información, 2012, septiembre-octubre, v. 21, n. 5, pp. 520-528.

http://dx.doi.org/10.3145/epi.2012.sep.12

\section{Introducción. El patrimonio audiovisual}

En la última década proyectos internacionales y autoridades en patrimonio audiovisual han advertido del riesgo de deterioro físico de las cintas de vídeo en formato analógico. El primer informe del proyecto PrestoSpace (Delaney; Hoomans, 2004) advertía de que los principales problemas con que se encontraban los archivos, bibliotecas, museos o instituciones culturales depositarios de estas colecciones, eran las deficientes condiciones de conservación que limitaban el acceso a los contenidos de los fondos audiovisuales. En muchos casos las dificultades se agravaban por el uso de equipos obsoletos y la falta de financiación.

El panorama no ha cambiado mucho en los últimos años. Richard Wright (2009), responsable de nuevas tecnologías de la cadena inglesa $B B C$, calcula que el $75 \%$ de los fondos audiovisuales mundiales tienen un acceso restringido y están en riesgo de desintegración física. Wright estima que sólo el $30 \%$ de los contenidos están sometidos a un plan de conservación. Este valioso patrimonio está amenazado por la obsolescencia de los formatos analógicos y el deterioro físico.

Consciente de este problema, en 2005 la Unesco declaró el 27 de octubre Día internacional del patrimonio audiovisual con el doble objetivo de hacer visible la necesidad de conservar unos documentos que forman parte de nuestra historia más reciente, y garantizar su transmisión a generaciones futuras.

Pero ¿cuáles son las dimensiones del reto de la preservación audiovisual? ¿Se ha podido cuantificar el volumen de este patrimonio? Una encuesta del proyecto Presto, de 20002001, estimó en 50 millones de horas los contenidos audiovisuales en toda Europa. Más adelante, en 2004 y 2005, PrestoSpace y especialmente TAPE, a partir de nuevas encuestas sobre más de 400 archivos, precisaron la cifra en 30 millones de horas. Por su parte en 2002 la Unesco calculó en más de 200 millones de horas el volumen del patrimonio audiovisual mundial (Wright, 2010).

Ante estas cifras PrestoPrime (Wright, 2010) plantea tres caminos básicos para salvar la información de los soportes magnéticos en formato analógico:

- conservar los originales en un ambiente frío, seco y estable;

- realizar copias cautelares utilizando la misma técnica o una parecida;

- migrar el contenido al entorno digital.

En el caso del patrimonio audiovisual, almacenado en cintas de vídeo, esta última opción parece ser la única viable. Hay que digitalizarlo para salvarlo, ya que todos los formatos analógicos han quedado obsoletos. Para la Association of
Moving Image Archivists (AMIA) (Videotape, 2007) la degradación de los soportes magnéticos es un hecho inevitable. Por ello las instituciones están obligadas a planificar estrategias para mejorar la esperanza de vida de las cintas, pero también a prever forzosamente planes de migración de los contenidos a nuevos soportes.

\section{La degradación de los soportes magnéti- cos es un hecho inevitable}

Los proyectos de digitalización de cintas magnéticas de vídeo son largos y costosos en términos económicos y de recursos humanos, lo que unido a los escasos recursos de tantas instituciones hace que muchas no puedan afrontar el problema de momento. A pesar de las dificultades, según las encuestas de Presto y PrestoSpace, se han digitalizado 4 millones de horas, sin contar las películas fílmicas. Esa cifra equivale a que cada año se está digitalizando el 1,5\% del patrimonio audiovisual estimado. Asumiendo que las principales colecciones audiovisuales tienen un volumen de 18 millones de horas y que el ritmo de digitalización es del 1,5\% anual, tras diez años de trabajo el triste resultado sería de 2,7 millones de horas migradas a codificación digital.

A ello hay que añadir que según el proyecto PrestoPrime, la ingesta de nuevos contenidos supone un aumento del $6 \%$ anual, cuatro veces superior a la tasa de digitalización. Según esto hay que tener en cuenta que:

- el 75\% de los 30 millones de horas de patrimonio audiovisual estimadas por PrestoSpace están en formato analógico y sólo el $25 \%$ ha sido digitalizado;

- la mayoría de los contenidos digitales están almacenados en cintas magnéticas, DAT o soportes ópticos;

- cada año se generan 75 petabytes de contenidos digitales en el mundo.

La mayoría de contenidos todavía están por digitalizar, con el riesgo de pérdida de información que conlleva. El tiempo juega en contra, pues el paso de los años dificulta la disponibilidad de un parque tecnológico de aparatos reproductores, su mantenimiento y encontrar de piezas de recambio. Además la mayoría de instituciones tienen como operadores a personal no especializado en el tratamiento de archivos audiovisuales en formato analógico.

Se estima que por cada hora de emisión es necesario destinar cuatro en digitalización y restauración. Los 30 millones de horas de patrimonio audiovisual en Europa se transformarían en 120 millones de horas de trabajo, o lo que es lo mismo, 13.700 años de una persona a tiempo completo.

Teniendo en cuenta estas magnitudes del trabajo y la inversión necesaria, es imprescindible que en las primeras etapas 


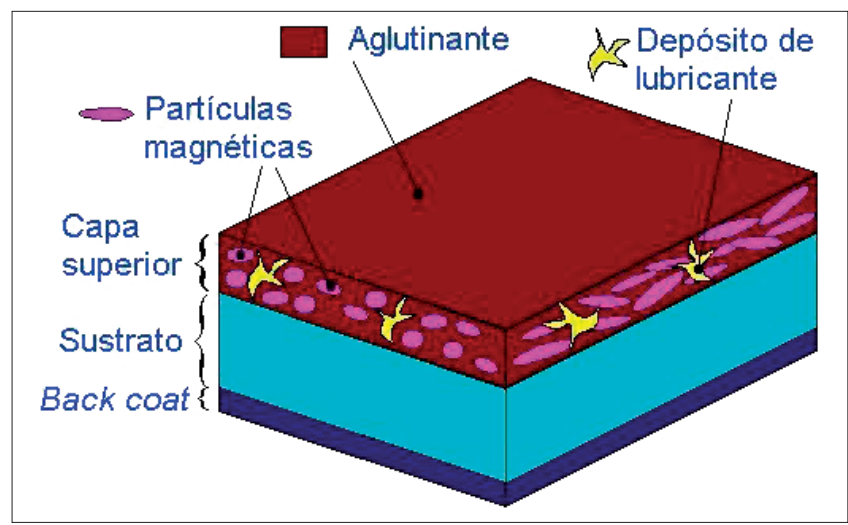

Figura 1. Capas de las cintas magnéticas. Fuente: Council on Library and Information Resources,

http://www.clir.org/pubs/reports/pub54/2what_wrong.html

de un proyecto de digitalización se realice una valoración correcta de los contenidos que realmente deben ser tratados. Como referencia sirvan los datos de una reciente estimación para la Comisión Europea (Poole, 2010), que indicaba que quizá sólo el $72 \%$ del patrimonio audiovisual europeo debería ser digitalizado; no cabe duda que esta proporción variará enormemente en cada colección concreta. La selección deberá estar a cargo de archiveros, con conocimiento del fondo y tratando de prever las necesidades futuras de los usuarios.

\section{Problemática de la conservación del vídeo analógico}

El adjetivo analógico describe el método usado para grabar información mediante una señal electrónica continua que altera el alineamiento de las partículas magnéticas de una cinta. Este procedimiento tiene la ventaja de su bajo coste pero supedita la longevidad de los contenidos a las fluctuaciones de los aparatos lectores y a la calidad del soporte donde están almacenados. El objetivo de los fabricantes de cintas de vídeo durante las décadas de 1970 y 1980 no era garantizar la preservación a largo plazo sino ofrecer un producto económico y accesible al consumidor doméstico.

Todas las cintas magnéticas presentan una estructura física similar compuesta por los siguientes elementos (figura 1):

- Base de la película: el soporte utilizado para construir este substrato es PET (Polyethylene terephthalate) o PEN (Polyethylene naphthalate), un tipo de poliéster con buenas propiedades como barrera en los procesos de descomposición. EI PET es un componente altamente estable que en un ambiente frío, seco y alejado de los rayos ultravioleta puede llegar a durar centenares de años. Ha sido comercializado en Estados Unidos y el Reino Unido con el nombre de Mylar.

- Recubrimiento magnético: constituido por una compleja mezcla de partículas magnéticas, adhesivo, lubricantes para reducir la fricción mecánica, agentes para limpiar los cabezales de lectura y otros productos químicos que pueden variar en función del fabricante.

- Adhesivo aglomerante de poliuretano o binder: añadido al recubrimiento magnético, sirve para unir las partículas magnéticas a la base de la película. Es el principal factor de degradación de las cintas.

- Revestimiento dorsal o backcoat: a finales de la década de 1960 la mayoría de las cintas de vídeo llevaban un revestimiento inferior compuesto de partículas de carbón sintético o carbon-black que cumplía una triple función: reducir la acumulación de electricidad estática cuando la cinta era reproducida a alta velocidad en el magnetoscopio; favorecer la unión de las capas de la cinta incrementando su durabilidad, y disminuir los roces mecánicos para protegerla de posibles arañazos.

- Partículas de óxidos metálicos: integradas en el adhesivo, pueden ser de dióxido de cromo u óxido de hierro. Las cintas de buena calidad además incorporaban una substancia antifúngica para prever la proliferación de mohos (figura 2).

Los soportes magnéticos están destinados a desaparecer con el paso del tiempo. Las condiciones ambientales en las que se almacenan son cruciales para determinar su esperanza de vida. Temperatura, humedad relativa del aire (HR), luz, presencia de agentes contaminantes y en menor medida existencia de campos magnéticos alrededor, tienen un papel importante en la preservación de las cintas de vídeo.

Toda la bibliografía técnica coincide en afirmar que la esperanza de vida de las cintas aumenta si se conservan en un ambiente frío, seco y con temperatura y humedad estables. La diversidad de opiniones empieza en el momento de establecer qué valores son más recomendables.

La esperanza de vida de las cintas aumenta si se conservan en un ambiente frío, seco y con temperatura y humedad estables

Algunos autores como Wheeler (2002) sostienen que la base de la película puede durar centenares de años si se conserva en las condiciones apropiadas. Nishimura (1991), investigador del Image Permanence Institute (perteneciente al Rochester Institute of Technology), añade que la duración del soporte está muy relacionada con el número de reproducciones, condiciones ambientales y calidad de los materiales en el proceso de fabricación de la cinta. El principal factor que condicionaría la esperanza de vida de una cinta de vídeo es la hidrólisis del poliuretano, que se usa como aglomerante por su flexibilidad. Para luchar contra este proceso es recomendable que las cintas se almacenen en un ambiente de humedad muy baja.

Si se conservan en ambientes con elevadas temperaturas y una HR alta el proceso de hidrólisis se acelera, con la contracción y expansión de la banda magnética, causando lesiones irreparables. El principal problema de conservación se centra en la perdurabilidad del aglutinante o adhesivo que une las partículas magnéticas con la base de la película.

La International Federation of Library Associations (IFLA, 1986) basa la estabilidad de los soportes magnéticos en una 
temperatura de 15 으, con una oscilación máxima de 3 으, y una HR inferior al 40\%. Esta misma institución recomienda que en ningún caso se superen los $23 \% \mathrm{C}$ de temperatura o el $70 \%$ de humedad. Las situaciones extremas de frío o calor aceleran los procesos de deformación de las bandas magnéticas y la aparición de moho.

Por otro lado, AMIA (Videotape, 2007) y el proyecto suizo Memoriav (2006) aceptan como buenas prácticas la conservación de cintas con base de poliéster a una temperatura de 20 으 y $20-30 \%$ de $\mathrm{HR}, 15$ 으 y $20-40 \%$ HR o 10 ㄷ C y $20-50 \%$ HR. Su recomendación para la preservación a largo plazo es de una temperatura de 8 으 y $25 \%$ HR con oscilaciones de $5 \%$ en la $\mathrm{HR}$ y $2 \stackrel{\circ}{ } \mathrm{C}$ en la temperatura.

A su vez el Image Permanence Institute (Adelstein, 2009) se adhiere a los planteamientos de la ISO 18923:2000 sobre cintas magnéticas de base de poliéster. Esta norma indica que en ningún caso la HR puede superar el $50 \%$ y establece una relación directa con la temperatura del depósito (ISO, 2000).

Los formatos analógicos han caído de forma fulminante en la obsolescencia tecnológica

Unas condiciones de almacenamiento inadecuadas pueden producir las siguientes patologías en los soportes magnéticos:

- Síndrome del vinagre o sticky shed syndrome: la descomposición del adhesivo provoca la presencia de una masa pegajosa en la superficie de la película que hace imposible su lectura. La cola afecta los cabezales del reproductor provocando la pérdida de señal o incluso que la máquina se atasque.

- Deformación del borde de las cintas o warped tape: provoca dificultades de reproducción y se produce por malas condiciones de almacenaje. A pesar de ello el poliéster es un material extremadamente resistente que en caso de deformación puede volver a su estado original si personal especializado con los conocimientos necesarios procede a su restauración.

- Mala señal de reproducción: se puede evitar limpiando las guías de las cintas, ajustando la tensión de la cinta o reproduciéndola en otro aparato lector. En algunos casos la pobre señal de reproducción puede ser debida a la reproducción de la cinta en un lector que no es compatible. Un ejemplo lo encontramos en las cintas de vídeo $s$-VHS que no son compatibles con los reproductores de VHS o los Quadruplex que pueden ser high band o low band.

- Desmagnetización: es poco habitual ya que se necesita un campo magnético muy potente para poder borrar el contenido de las cintas. Los campos magnéticos de televisores, transformadores o motores son demasiado débiles para provocar daños en las cintas de vídeo. Se recomienda evitar la exposición prolongada a altas temperaturas que pueden provocar pequeños porcentajes de desmagnetización. Una temperatura de 10 ㄷ C y una HR del $60 \%$ concede una esperanza de vida de 700 años a las partículas metálicas de las cintas.

\section{La digitalización como vía de preservación}

Las principales marcas comerciales determinan la longevidad de una cinta magnética en 25 años si se ha conservado en unas condiciones ambientales estables. Por otro lado, para evitar pérdidas de información el proyecto TAPE (Schüller, 2008) aconseja migrar el contenido de una cinta analógica a un formato digital en un período máximo de 20 años. Podemos afirmar pues que los formatos analógicos han caído de forma fulminante en la obsolescencia tecnológica.

Las dificultades no están solo en la supervivencia de las cintas magnéticas. Hay que añadir el problema de mantener un parque tecnológico de magnetoscopios que han desaparecido de los circuitos comerciales y que los fabricantes han dejado de fabricar (figura 3). La falta de aparatos lectores puede hacer imposible recuperar la información de su interior. Sólo el control de las condiciones ambientales no garantiza que las cintas se puedan reproducir en el futuro.

La preservación digital es un reto enorme para la mayoría de archivos, bibliotecas, museos o instituciones que custodian el patrimonio audiovisual. Gestionar sus activos requiere una gran inversión de tiempo, un enorme esfuerzo del personal y elevados costes económicos. Jones y Beagrie (2002) definen la preservación digital como "la serie de actividades necesarias para garantizar el acceso continuo a los materiales digitales durante el tiempo que sea necesario [...] más allá de los límites, errores, medios y cambios tecnológicos».

La preservación digital a largo plazo pretende conservar un fichero máster lo más próximo posible a la fuente analógica original. La estrategia de preservación de este máster digital debe incluir la comprobación de la integridad de los datos, su restauración en caso de ser necesario y la migración a futuras plataformas tecnológicas para evitar la obsolescencia, con el mínimo de pérdida de información en el proceso. La fidelidad con el original a menudo supone el uso de formatos digitales muy ricos que requieren una gran capacidad de almacenaje, uno de los principales problemas de los ficheros digitales de vídeo.

Para llevar a cabo estos proyectos de digitalización son necesarios técnicos especialistas con habilidades y conocimientos específicos en el tratamiento de fondos audiovisuales. Según la encuesta desarrollada por el proyecto TAPE (2007), el $61 \%$ de los centros depositarios del patrimonio audiovisual confesaba no tener el personal adecuado y el $38 \%$ reconocía serias dificultades para recibir formación en su país.

\section{Características técnicas del vídeo digital}

Conocer las características formales del vídeo digital, los formatos existentes y su estructura interna es imprescindible para una buena toma de decisiones en un proyecto de digitalización pensando en la preservación a largo plazo.

La mayoría de los formatos de vídeo digital, responden a una estructura común formada por cuatro capas: imagen móvil, datos del audio, el encabezamiento que contiene la información necesaria para descodificar y los metadatos para el flujo de información. 
Por otra parte existen las características formales de los ficheros de vídeo que definen la imagen y sus niveles de calidad. En función de las características formales escogidas la calidad final puede variar. A continuación se detallan los principales parámetros a configurar para la conversión de una cinta magnética de vídeo con señal analógica a un formato de archivo digital:

- Sistema de codificación: los principales sistemas analógicos de codificación y transmisión de la señal televisiva en color son NTSC, PAL y Secam. Difieren en el número de fotogramas por segundo utilizado y el número de líneas de la imagen entrelazada. La calidad de la imagen reproducida es directamente proporcional al número de líneas por pantalla, siendo necesario un número mínimo para que la trama no se visualice demasiado.

- Relación de aspecto: proporción entre alto y ancho de la pantalla. El 4:3 fue en el pasado el estándar televisivo más habitual, pero actualmente los televisores panorámicos de alta definición utilizan 16:9.

- Imágenes por segundo o frame-rate: velocidad a la que pasan las imágenes por la pantalla, equivalente al número de fotogramas mostrados por segundo. Se mide en $f p s$ o frames per second y varía en función de los diferentes estándares televisivos: el cine de dibujos animados utiliza una framerate de $15 \mathrm{fps}$, PAL de 25 fps y NTSC de 29,97 fps.

- Modo de exploración: forma en que el monitor escanea la rápida sucesión de imágenes que generan la sensación de movimiento. Los principales son el escaneo entrelazado (líneas $1,3,5 \ldots$, seguidas de $2,4,6 \ldots$ ) y el sistema progresivo (líneas 1, 2, 3, 4...). Si el ancho de banda no es un problema, pues se necesita el doble, la calidad del modo progresivo es netamente superior.

- Resolución: número de píxeles que pueden ser mostrados por pantalla. Se indica con el número de píxeles de alto por el número de píxeles de ancho que tiene la imagen. En la digitalización, la resolución debe estar de acuerdo con la calidad del original.

- asa de bits o bitrate: importante para definir el volumen y la calidad final del fichero de vídeo digital. Indica el número de bits por unidad de tiempo que se transmite entre dos dispositivos, su unidad de medida son los kilobits por segundo (Kbps).

Para ahorrar costes de almacenaje y facilitar la transmisión de los datos, algunos formatos de vídeo digital aplican técnicas de compresión de datos para reducir su volumen, eliminando los elementos redundantes e irrelevantes. Los codecs son algoritmos matemáticos de comprimir y descomprimir esta información facilitando la reproducción. Básicamente existen dos sistemas de compresión:

- Sin pérdida de información. Los datos de salida del decodificador son idénticos a la fuente original. Dance Heritage Coalition (Digital, 2004) con la frase "There is no free lunch", indica que plantea dos problemas principales: la gran cantidad de tiempo y la capacidad de procesamiento necesario para comprimir los ficheros, y los pobres ratios de compresión logrados.

- Con pérdida de información. Parte de la información se ha eliminado intencionadamente para ahorrar costes de almacenaje y transmisión. El fichero resultante puede presentar imágenes de suficiente calidad para engañar a nuestros ojos y que no noten la diferencia con la fuente original; es la denominada "compresión subjetivamente sin pérdidas". Cuando la compresión es máxima y las pérdidas de información se perciben pero son tolerables hablamos de "compresión subjetivamente con pérdidas". Es el caso de los vídeos de YouTube o las copias en baja resolución para publicar en la Web.

\subsection{Contenedores multimedia (wrappers)}

Formato de archivo que almacena información de vídeo y audio (Ilamada a veces esencia, essence), subtítulos, metadatos y otras informaciones.

Partiendo de la premisa de que es necesario capturar la señal analógica de las cintas de vídeo con la mejor calidad posible y en función de la fuente original, hay un consenso profesional en reclamar la utilización de estándares de código abierto. Del mismo modo se aconseja que el formato contenedor sea de uso generalizado en el mercado y se pueda abrir, leer y acceder utilizando herramientas de fácil disponibilidad. A continuación reseñamos brevemente los contenedores multimedia con más presencia en el mercado audiovisual, siempre desde la perspectiva de la preservación a largo plazo de los contenidos:

\section{Audio video interleave o $\mathrm{AVI}$}

Formato de archivo propietario de vídeo y audio desarrollado por Microsoft en noviembre de 1992. Permite almacenar un flujo de datos de vídeo y varios de audio, pero para que estos flujos de datos se puedan reproducir al mismo tiempo es necesario que se almacenen de forma entrelazada, de aquí el nombre de interleave. Utiliza codecs de compresión ya que si se almacenara sin comprimir puede llegar a ocupar una memoria de $30 \mathrm{~GB} /$ hora de grabación. Los codecs de vídeo más utilizados son el JPEG 2000, MPEG, Indeo, DV, DivX o Cinepack. En el caso de las pistas de audio los codecs habituales son el WAVE, MP3 CBR, AC3, DivX audio o DTS.

\section{Flash video o FLV}

Formato propiedad de Adobe Systems. Utiliza codecs de compresión de audio y vídeo que en la mayoría de los casos han sido patentados por empresas privadas. El uso de Flash video ha tenido una gran aceptación y, desde su lanzamiento en 2002 , se calcula que el $96 \%$ de los equipos conectados a internet lo utiliza habitualmente.

\section{Material exchange format o MXF}

Formato profesional de código abierto. Surgió como una solución a la poca interoperabilidad entre plataformas de edición y dispositivos de creación de contenidos en los entornos de producción profesional. Permite guardar e intercambiar datos de vídeo y audio con sus metadatos en la cabecera del contenedor, mejorando la gestión de la información asociada a las imágenes.

\section{Matroska o MKV}

Formato de código abierto y libre de patentes que aspira a convertirse en el formato universal para el almacenaje de contenidos multimedia. Es una alternativa a los forma- 
tos propietarios como AVI, ASF, MOV o MPEG. Destaca por su flexibilidad y capacidad para almacenar información de audio, vídeo y subtítulos ocupando menos espacio de disco que AVI. Entre sus inconvenientes está que es de creación algo reciente (2003) y muchos usuarios por desconfianza o ignorancia apuestan todavía por formatos propietarios, pero es posible que esta tendencia vaya cambiando con el paso del tiempo. Le hace falta una mayor compatibilidad con los programas reproductores de vídeo.

\section{Moving picture expert group o MPEG}

Es uno de los formatos más utilizado en el ámbito doméstico por su compatibilidad con la mayoría de reproductores DVD de sobremesa. Recientemente su hegemonía se ha visto cuestionada por la aparición en el mercado de codecs como el Xvid o Dvix. Es capaz de alojar una pista de vídeo utilizando una codificación MPEG-1 o MPEG-2 y diversas pistas de audio en formato WAV, MPEG o Dolby Digital AC3.

\section{QuickTime file format}

Desarrollado por Apple. Los archivos QuickTime utilizan la extensión .mov y son compatibles con los sistemas operativos MAC OS, MAC OS X y Microsoft Windows pero todavía no existe ninguna versión disponible para Linux. El 98\% de los usuarios que se descargaron la sexta versión era usuarios de PC, a un ritmo de 10 millones de descargas por mes. Está pensado para reproducir vídeo a través de internet. Con mil millones de descargas, su software es líder de la industria de desarrollo, producción y entrega de vídeo y audio de alta calidad por IP.

\subsection{Estándares de compresión de vídeo digital}

Las técnicas de compresión de vídeo digital se centran en intentar reducir al máximo la cantidad de información necesaria para transmitir una secuencia de imágenes, respetando en la medida de lo posible la calidad. Los codecs de vídeo con más presencia en el mercado y sus propiedades más destacadas son los siguientes:

- Digital video o DV: diseñado en la década de 1990, inicialmente fue concebido como un formato de vídeo digital profesional, pero su excelente relación calidad-precio lo convirtió en el gran dominante del mercado doméstico con el formato Mini DV. Su popularidad va más allá del gran consumo y existen versiones profesionales. Puede ser encapsulado en contenedores multimedia como AVI, MXF o QuickTime.

- H.264 / MPEG-4 AVC: estándar de codificación de vídeo resultado del trabajo conjunto de dos instituciones: la International Standards Organization (ISO) y la International Telecommunications Union (ITU). Formalmente conocido por ISO/IEC 14496-10:2003 es un estándar de compresión abierto que reduce la tasa de transferencia de bits sin incrementar la complejidad del diseño o disminuir la calidad de la imagen. Ha sido adoptado por mercados tan diferentes como las videoconsolas, los discos de alta definición Blu-ray o el Ipod.

- Motion Picture Expert Group-2 o MPEG-2: formalmente denominado ISO/IEC 13818, es un estándar de compresión vídeo con pérdidas que analiza la diferencia entre fotogra-

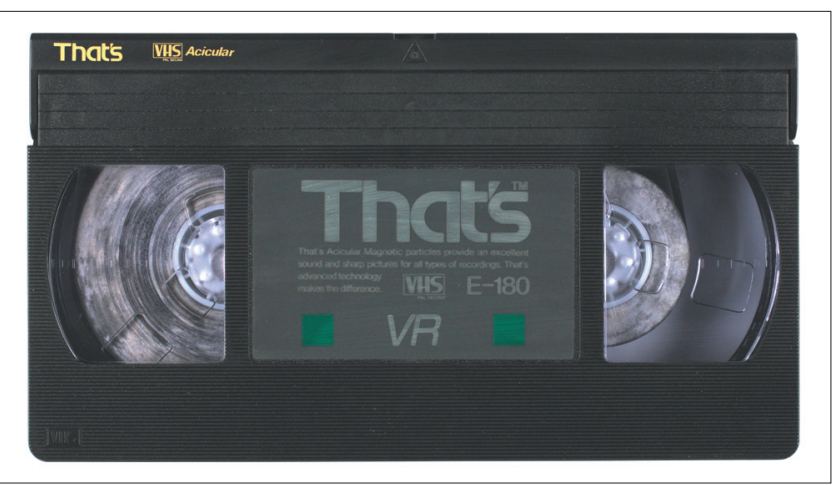

Figura 2. Muestra de una cinta magnética en formato VHS con moho debido a un almacenaje en condiciones ambientales inapropiadas

mas consecutivos para reducir la tasa de transferencia de bits. A pesar de la amplia aceptación que tiene en el mercado audiovisual, especialmente por su adopción como sistema de compresión de vídeo digital en DVD, algunos autores consideran que está obsoleto y que su uso disminuirá en beneficio de otros codecs más eficientes.

- OGG Theora: códec de vídeo de código abierto que forma parte del proyecto sin ánimo de lucro OGG. Aplica un algoritmo de compresión con pérdidas que puede almacenarse dentro de cualquier formato contenedor multimedia y normalmente se combina con el códec de audio Vorbis. Disponible desde noviembre de 2008, es ideal para difundir vídeo en streaming por internet.

- VC-1 o Smpte 421: la Society of Motion Picture and Television Engineers (Smpte) publicó las especificaciones técnicas de este estándar abierto en abril de 2006. Es la culminación del trabajo de más de 75 empresas para dotar a la industria audiovisual de un códec estable, accesible, bien documentado, aceptado en el mercado y con una licencia fácil de adquirir. Además, Windows Media Video 9 o WMV9 es la implementación que ha hecho la multinacional Microsoft del estándar VC-1. A fecha de hoy soportes como el HD DVD o el Blu-ray lo han adoptado como un estándar de vídeo obligatorio y ha seducido a la industria audiovisual por su calidad y escalabilidad.

\subsection{Estándares de compresión de audio digital}

El audio digital está formado por una reproducción de las ondas que al vibrar generan el sonido. Estas formas de onda son muestreadas a intervalos de tiempo específicos y cuantificadas con valores binarios.

A continuación se citan de forma sumaria los codecs de audio con más presencia en el mercado y sus principales especificaciones técnicas. La información se basa en el excelente estudio de sostenibilidad de formatos digitales de la Library of Congress de los EUA (Library of Congress, 2009):

- Advanced audio coding o AAC: códec de código abierto que utiliza un algoritmo de compresión con pérdidas. Desarrollado por el grupo MPEG, ha sido estandarizado por la norma ISO/IEC 13818-7 como parte de las especificaciones de MPEG-2 y MPEG-4. Tiene una fuerte presencia en el mercado al ser escogido por Apple como el formato principal para iPods, iPads, iPhones y el software iTunes, además de algunas videoconsolas. 
- Audio interchange file format o AIFF: estándar de audio desarrollado por Apple en 1988. El hecho de ser un formato de compresión sin pérdidas lo convierte en apto para la preservación a largo plazo, pero presenta como inconveniente la gran capacidad de almacenaje que necesita: $10 \mathrm{MB}$ equivalen a 1 minuto de audio estéreo con una frecuencia de muestreo de $44,1 \mathrm{KHz}$ y 16 bits de profundidad. Es uno de los formatos de audio más aceptados por el mercado.

- Free lossless audio o FLAC: códec de audio de código abierto, libre de patentes y que aplica una compresión de datos sin pérdidas. Desde 2003 forma parte del proyecto de Xiph.org Foundation junto a otros codecs como Vorbis o Theora. Sus propiedades lo convierten en ideal para la preservación a largo plazo pero su implementación en el mercado todavía es limitada en comparación con otros formatos.

- MPEG-1 audio layer 3 o MP3: códec de audio digital que aplica algoritmos de compresión con pérdidas de información. Actualmente goza de una gran aceptación en el mercado de la transmisión y reproducción de audio digital por lo que se ha convertido en un estándar de facto. A pesar de ello, los elevados ratios de compresión no lo hacen aconsejable para la preservación a largo plazo.

- Wave audio format o WAVE: estándar de compresión de datos de audio sin pérdidas de información propiedad de las multinacionales Microsoft e IBM. Es el formato de audio más utilizado por el sistema operativo Windows y también opera con Macintosh y Linux. A pesar de las grandes dimensiones de sus ficheros ha sido adoptado para la preservación a largo plazo por algunas emisoras como BBC Radio o Australian Broadcasting Corporation.

\section{Adecuación de las características técnicas a las necesidades del servicio}

Para minimizar los costes de la gestión se aconseja aprovechar al máximo los recursos propios disponibles y apostar por el desarrollo del proyecto de digitalización en la misma institución. Según el proyecto TAPE (Pavuza, 2008), sólo sería recomendable la subcontratación a empresas especializadas cuando los formatos analógicos de cinta tengan muy poca presencia en el mercado y sea muy difícil conseguir aparatos lectores. Los servicios de hosting tampoco son aconsejables por su elevado coste y la dificultad de transmisión de los datos con ficheros de grandes dimensiones. En todo caso, a la hora de decidir la posible externalización de las operaciones será de gran importancia tener en cuenta el volumen de fondos a tratar.

Aun así, los centros deben prever elevadas inversiones económicas en hardware y software, necesarias para la captura de los contenidos, el almacenaje y gestión de los ficheros digitales. Otro paso imprescindible es proveerse de un parque de aparatos lectores capaces de leer todos los formatos de cinta analógica existentes. Si el centro ya dispone de una parte o la totalidad del hardware sólo deberá comprobar su funcionamiento, pero si no es así, su localización puede ser una ardua tarea debido a su escasez en el mercado.

Entre las habilidades del personal propio que ejecute o supervise el proyecto de digitalización no pueden faltar:
- capacidad de identificar los diferentes formatos de cintas magnéticas;

- conocimiento del funcionamiento del software de gestión y el parque tecnológico;

- capacidad de detectar las patologías que sufren las cintas con una inspección sensorial;

- conocimientos actualizados sobre estudios en materia de preservación digital.

La elección del formato contenedor de vídeo digital que se utilizará para almacenar la señal de vídeo analógico capturado es un aspecto crucial del proyecto. El propósito inicial debe ser realizar una conversión de formato obteniendo un fichero digital lo más fiel posible al original. Este principio fundamental de la archivística queda recogido en definiciones tales como la elaborada por el Coordinating Council of Audiovisual Archive Association (Unesco, 2005) sobre el concepto de preservación: "medidas necesarias para garantizar la accesibilidad permanente de un documento audiovisual con la máxima integridad".

Actualmente la digitalización de la señal analógica de vídeo sin comprimir puede generar ficheros digitales con un peso aproximado de $70 \mathrm{~GB} /$ hora. Los costes de los dispositivos de almacenaje pueden resultar prohibitivos para instituciones de pequeñas y medianas dimensiones.

Si el vídeo analógico se captura sin compresión o una compresión sin pérdidas se obtendrán ficheros de grandes dimensiones que en muchos casos hipotecarán los recursos del proyecto. Pero si la digitalización utiliza codecs de compresión con pérdidas, parte de la información se perderá irremediablemente y no se podrá recuperar sin repetir el proceso de digitalización, con los nuevos costes que ello implica. Por tanto, la elección del formato contenedor y los codecs de audio y vídeo es una de las decisiones más difíciles e importantes del proyecto, y tomarse en función de los objetivos y recursos disponibles, adoptando la solución más adecuada para los propósitos de la institución.

La preservación a largo plazo de los archivos audiovisuales implica un proceso continuo de migraciones a nuevos soportes y formatos

Para Schüller (2009), director de Phonogrammarchiv, las políticas de digitalización de las televisiones y las instituciones culturales no tienen por qué ser las mismas. Los archivos televisivos persiguen unos fines empresariales que justificarían apostar por formatos de archivo digital que apliquen ratios de compresión con pérdidas como el códec MPEG-2, generalmente a un bitrate de $50 \mathrm{Mbps}$. Instituciones como archivos, bibliotecas y museos tienen el deber ético de intentar seguir una política diferente basada en la apuesta por formatos de archivo sin comprimir, preservando en las mejores condiciones posibles toda la información a su cargo. Esta tesis puede verse favorecida por la constante disminución de los costes de almacenaje y el desarrollo comercial de herramientas de software y hardware especialmente pensadas para tareas de archivo. 


\section{Conclusiones}

La preservación del patrimonio audiovisual no es un reto fácil. Estamos ante un escenario de incertidumbre entre la esperanza de vida de los formatos analógicos y el cambio de paradigma tras la aparición de la tecnología digital. Nos preguntamos si esta nueva realidad supone una transición o una ruptura en el proceso de transformación de la imagen. Unos cambios que a su vez son reflejo de las transformaciones económicas y sociales de nuestro entorno.

La conversión de vídeo de analógico a digital supone unos elevados costes de almacenaje. Por ejemplo, una hora de vídeo ocupa 150 veces más memoria digital que el audio. De este modo lo que en la digitalización de audio puede ser una opción asequible, para el vídeo se convierte en una decisión difícil. A ello hay que sumarle que no existe un formato de archivo estándar de vídeo como sería la apuesta por el formato WAVE en el audio digital.

Debemos tener en cuenta que si las dificultades técnicas, de presupuesto y personal frenan el desarrollo del proyecto quizá cuándo la institución esté preparada para intervenir ya no queden contenidos que salvar. Así pues hay que actuar, pero para Wright (2009) toda intervención debería guiarse por el siguiente principio general: si el formato de archivo digital escogido aplica un códec de compresión con pérdidas, obligado por posibles limitaciones presupuestarias del proyecto, bajo ningún concepto se debe migrar en el futuro a un formato digital diferente que también aplique compresión con pérdidas, pues en el proceso de recodificación lo más probable es que la nueva generación digital tenga una gran pérdida de calidad.

Tras la digitalización surge la necesidad de conservar y garantizar el acceso a los nuevos contenidos digitales, más inestables si cabe que los primeros. La incesante evolución de los mercados y el paso del tiempo se avecinan como los principales obstáculos a superar. Si antes hablábamos de un período de 20 años para migrar los contenidos de las cintas de vídeo analógico a digital, las perspectivas actuales aconsejan migrar los datos digitales cada 5 ó 10 años para garantizar su legibilidad. Estudios recientes de Google calculan que la supervivencia de los soportes físicos de almacenaje, concretamente los discos duros magnéticos, no va más allá de los 5 años (Pinheiro; Weber; Barroso, 2007).

Entonces podemos preguntarnos ¿por qué digitalizar una señal analógica de vídeo si la digital está igualmente sometida al deterioro físico del soporte de almacenaje y a la obsolescencia tecnológica? La respuesta es muy sencilla, el soporte es un elemento efímero que no determina la naturaleza de la imagen. La preservación a largo plazo de los archivos audiovisuales siempre implicará un proceso continuo de migraciones de los contenidos a nuevos soportes y formatos.

Afortunadamente, los centros de patrimonio audiovisual no están solos en este desafío. La creación de contenidos digitales se ha extendido a muchos ámbitos de nuestra sociedad y ha surgido un mercado en la necesidad de conservarlos. A su vez, proyectos internacionales en activo como PrestoPrime son los encargados de liderar las iniciativas sobre preservación a largo plazo de los archivos audiovisuales,

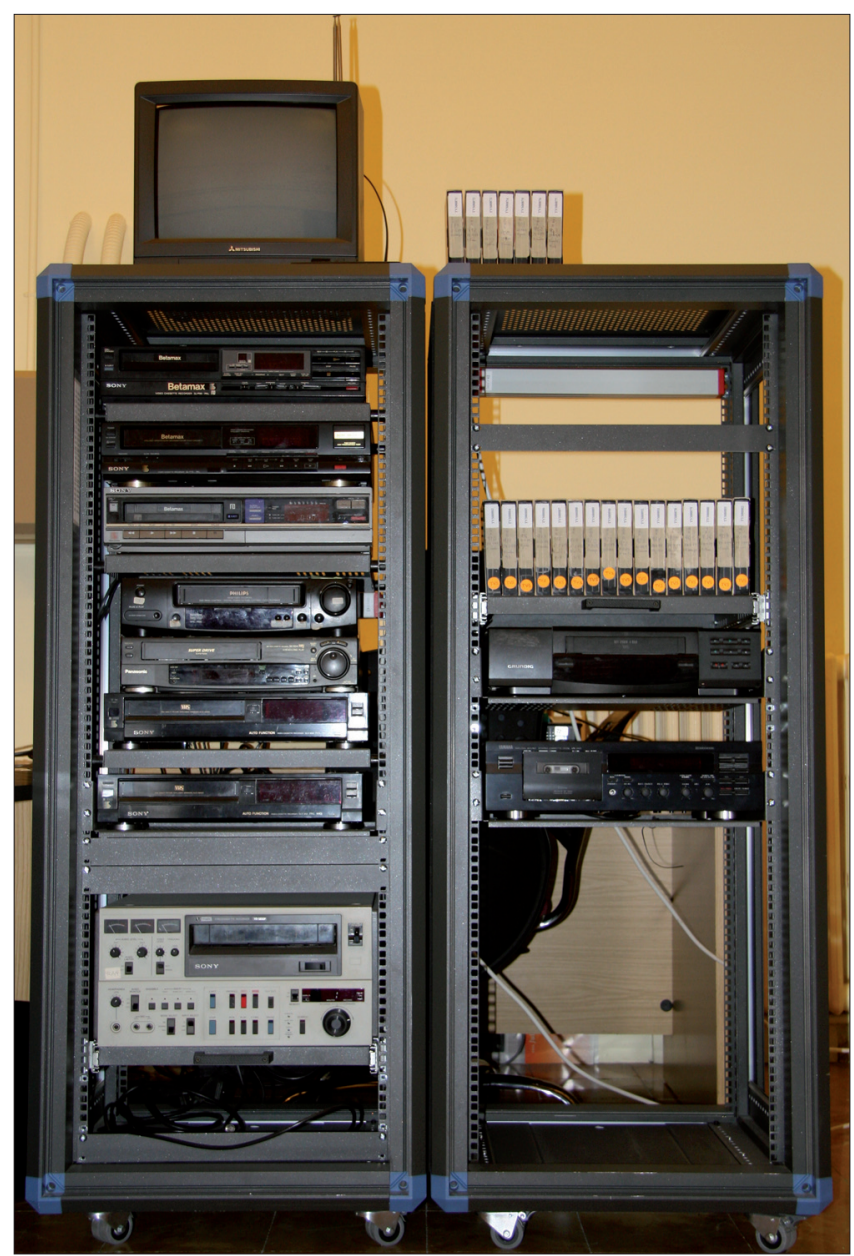

Figura 3. Aparatos lectores VHS, Betamax y U-matic del Archivo Histórico de Sabadell

reuniendo los profesionales más cualificados del sector y profundizando en el estudio de las directrices a seguir.

\section{Bibliografía}

Adelstein, Peter Z. IPI media storage quick reference. Rochester: Image Permanence Institute, 2009.

http://www.imagepermanenceinstitute.org/shtml_sub/ MSQR.pdf

Delaney, Beth; Hoomans, Brigit. Requeriments final report. PrestoSpace Questionnaire 1. PrestoSpace, 2004.

http://prestospace.org/project/deliverables/D2-1_User_ Requirements_Final_Report.pdf

Digital video preservation reformatting project. A report. Dance Heritage Coalition, 2004.

http://www.media-matters.net/docs/DigitalVideoPreserva tion1[1].pdf

IFLA. Principles for the care and handling of library material. International Federation Library Associations, 1986. http://archive.ifla.org/VI/4/news/pchlm.pdf

ISO 18923:2000. Imaging materials - Polyester-base magnetic tape - Storage practices. Ginebra: International Organization for Standardization, 2000.

Jones, Maggie; Beagrie, Neil. Preservation management of digital materials: a handbook. London: British Library, 2002. 
http://www.dpconline.org/pages/handbook/index.html

Library of Congress. "Format descriptions for sound". Sustainability of digital formats. Planning for Library of Congress collections. Washington DC: Library of Congress, 2009.

http://www.digitalpreservation.gov/formats/fdd/sound_ fdd.shtml

Memoriav recommandations video. La sauvegarde de documents video. Berne: Memoriav, 2006.

http://fr.memoriav.ch/dokument/Empfehlungen/empfe hlungen_video_fr.pdf

Nishimura, Doug. "Stability of videotape and optical disks". Conservation DistList, 19 mayo 1991.

http://cool.conservation-us.org/byform/mailing-lists/ cdl/1991/0146.html

Pavuza, Franz. Short guidelines for video digitization. Training for audiovisual preservation in Europe, 2008.

http://www.tape-online.net/Short_Guidelines_Video_ Digitisation.pdf

Pinheiro, Eduardo; Weber, Wolf-Dietrich; Barroso, LuizAndré. "Failure trends in a large disk drive population". En: $5^{\text {th }}$ Usenix conf. on file and storage technologies (FAST'07), Berkeley, 2007.

http://static.googleusercontent.com/external_content/un trusted_dlcp/research.google.com/ca//archive/disk_failu res.pdf

Poole, Nick. The cost of digitising Europe's cultural heritage. A report for the 'comité des sages' of the European Commission. November 2010, 79 pp.

http://ec.europa.eu/information_society/activities/digital_ libraries/doc/refgroup/annexes/digiti_report.pdf

Schüller, Dietrich. Audiovisual research collections and their preservation. European Commission on Preservation and Access, 2008.

http://www.tape-online.net/docs/audiovisual_research_ collections.pdf

Schüller, Dietrich. "Video archiving and the dilemma of data compression". Intl. preservation news, 2009, n. 47, pp. 5-7. http://www.ifla.org/files/pac/IPN_47_web.pdf

Tape survey factsheet. Training for audiovisual preservation in Europe, 2007.

http://www.tape-online.net/docs/Tape_survey_factsheet.pdf

Unesco instrument for the safeguarding and preservation of the audiovisual heritage: CCAAA issues paper. Coordinating Council of Audiovisual Archive Association, 2005. http://www.ccaaa.org/docs/ccaaa_heritage.pdf

Videotape preservation fact sheets. Hollywood: Association of Moving Image Archivists, 2007.

http://www.amianet.org/resources/guides/fact_sheets.pdf

Wheeler, Jim. Videotape preservation handbook, 2002.

http://www.media-matters.net/docs/resources/Traditional Audiovisual Preservation/WheelerVideo.pdf

Wright, Richard. "Audiovisual digital preservation status report”. En: PrestoPrime, Viena, 2010.

https://prestoprimews.ina.fr/public/deliverables/PP_WP7_ D7.1.3_Annual_AV_Status_R0_v1.00.pdf

Wright, Richard. "Digital preservation strategies". En: PrestoPrime, Viena, 2009.

http://www.prestoprime.org/training/index.en.html

Wright, Richard. "Preservation of broadcast archives: a BBC perspective". International preservation news, 2009, n. 47, pp. 13-17.

http://www.ifla.org/files/pac/IPN_47_web.pdf

\section{Si te interesan los}

\section{INDIGADORES EN TENGIA A ENOLOGÍA,}

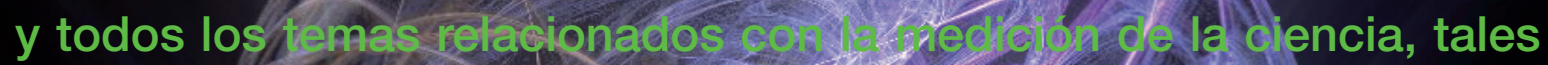
como:

Análisis de citas, Nomaliractus s. istuciones, Impacto de la ciencia en la socecrar. ores) Sociología de la ciencia, Política científica, Cophoiguarón o la clencla, Revistas, Bases de datos, Índices de impacto, ol ticjact de open access, Análisis de la nueva economia, Mujer,y, siencia,

\section{Entonces ING es tu lista. Suscribete en:}

\section{http://www.rediris, es/list/info/incyt.htmI}

